\title{
Radiation-induced Errors in Memory Chips
}

\author{
R. J. Peterson \\ University of Colorado, \\ Boulder, CO 80309-0446 USA
}

Received on 30 October, 2002

\begin{abstract}
We have measured probabilities for proton, neutron and pion beams from accelerators to induce temporary or soft errors in a wide range of modern $16 \mathrm{Mb}$ and $64 \mathrm{Mb}$ DRAM memory chips, typical of those used in aircraft electronics. Relations among the cross sections for these particles are deduced. Measurement of alpha particle yields from pions on aluminum, as a surrogate for silicon, indicate that these reaction products are the proximate cause of the charge deposition resulting in errors.
\end{abstract}

\section{Introduction}

In 1978 a serious industrial problem was causing errors in the memory contents of Intel 2107 16Kb DRAM (Dynamic Random Access Memory) chips.[1] These temporary or soft errors did not damage the chip, but an erroneous 'zero' had replaced a 'one', or vice versa, spoiling the contents of the memory. This problem was traced to small amounts of alpha-emitting impurities in the materials. These $5 \mathrm{MeV}$ alpha particles caused ionization in the Si of the chip, and the collected charge was enough to redefine the memory state.

Later, in repair records from 1984, IBM found a correlation between these errors, here called 'soft error upsets' or SEU, and altitude. These errors were then shown to be closely correlated with cosmic ray intensities.[2, 3] In Denver, at $1700 \mathrm{~m}$, the SEU rate was ten times the national average.

It is easy to understand how heavily-ionizing low energy alpha particles, with a range of 25 microns in $\mathrm{Si}$, could deposit disruptive charge, but how would high energy neutrons, protons and pions from cosmic ray reactions induce errors? At sea level, most cosmic rays are muons, with only small cross sections for any reactions, but evidently energetic hadrons can cause reactions within microcircuits to produce short range heavily ionizing reaction products.

A series of experiments with intense accelerator beams has been used to measure the probabilities or cross sections for hadrons to induce SEU in a wide range of modern memory chips. These measurements are particularly important for commercial and military aircraft, with their high altitude operations.

Here we define

$$
\sigma_{S E U}=\frac{\text { induced errors }}{\text { number of sample bits } \times \text { particles } / \mathrm{cm}^{2}} .
$$

\section{Accelerator testing}

Commercially available memory chips were placed directly in our beams. Proton beams were mostly from the Harvard cyclotron, at energies from 50 to $150 \mathrm{MeV}$.[4] Pion beams from LAMPF [5] and TRIUMF [6] and a $14 \mathrm{MeV}$ neutron generator [7] have been used. Appropriate beam monitoring gave beam fluxes, for instance $0.1-1 \times 10^{6} \mathrm{pions} / \mathrm{cm}^{2} \mathrm{sec}$.

A special driver box alternately wrote and read patterns of zeros and ones into the memory chips. Discrepancies were counted as errors. The $16 \mathrm{Mb}$ samples used three technologies - Trench External Charge (TEC), Stacked Capacitor (SC) and Trench Internal Charge (TIC). Samples from several manufacturers were chosen for TEC and SC technologies.

For $16 \mathrm{Mb}$ TEC samples the pion cross sections showed clear evidence, in the macroscopic chip, of the familiar 33 pion-nucleon resonance.[5] See Fig. 1. We also note the expected charge symmetry between $\pi^{+}$and $\pi^{-}$beams for reactions upon symmetric ${ }^{28} \mathrm{Si}$. The curve shows the computed $\pi^{+} / \pi^{-}$averaged ${ }^{28} \mathrm{Si}$ reaction cross section.[8] Proton SEU cross sections are lower, and show no resonant effect; proton reaction cross sections show no resonances in this high range of energies.[9] A single datum shows the SEU cross section for $14 \mathrm{MeV}$ neutrons. A smaller range of pion energies was used for the $64 \mathrm{Mb}$ DRAM samples, and the 3-3 resonance was not obvious, as seen in Fig. 1.[6]

\section{Comparisons}

SEU cross sections for $16 \mathrm{Mb}$ samples showed a very wide range, strongly dependent upon the chip construction. Cross sections are shown in Fig. 2. For some manufacturers, several different chips of the same model were studied, with little scatter. The large differences can be explained by the smaller volume of $\mathrm{Si}$ available to deposit stray charge in the newer generations. 


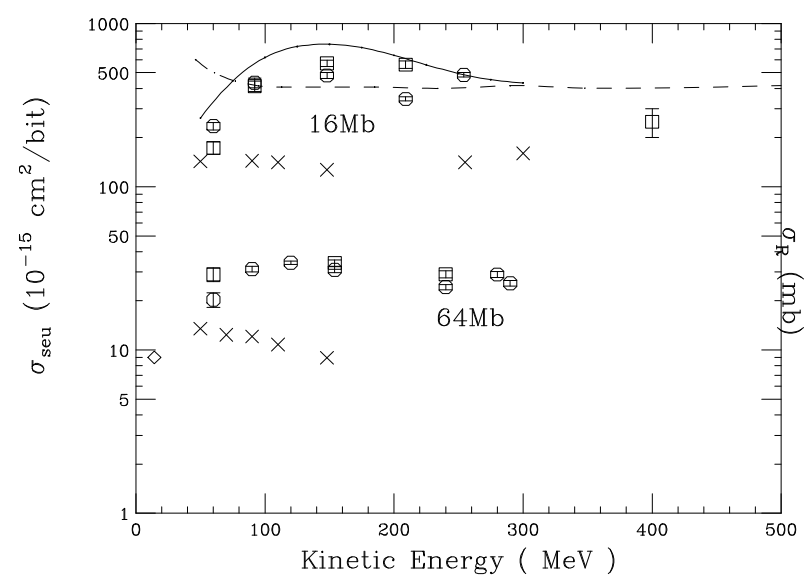

Figure 1. Crosses show measured cross sections for protoninduced SEU in $16 \mathrm{Mb}$ and $64 \mathrm{Mb}$ DRAM samples. The $16 \mathrm{Mb}$ sample used the TEC technology. Squares show data for $\pi^{-}$, circles for $\pi^{+}$for the same chip. The solid curve shows computed [8] $\pi^{+} / \pi^{--}$averaged reaction cross sections for ${ }^{28} \mathrm{Si}$ using the right hand scale. The pi-nucleon 3-3 resonance is prominent in the 16 $\mathrm{Mb}$ macroscopic device. The dashed curve shows proton reaction cross sections.[9] A single datum shows the cross section for 14 $\mathrm{MeV}$ neutrons.

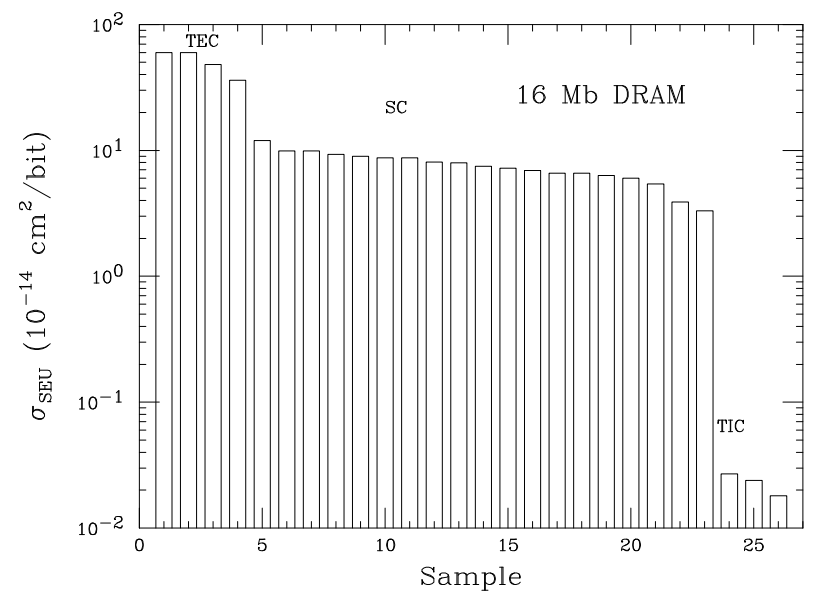

Figure 2. Cross sections for SEU for many samples of $16 \mathrm{Mb}$ dRAM chips are compared for $150 \mathrm{MeV} \pi^{+}$beams. TEC, SC and TIC samples are clearly separated. Examples with the lowest cross sections use the TIC technology.

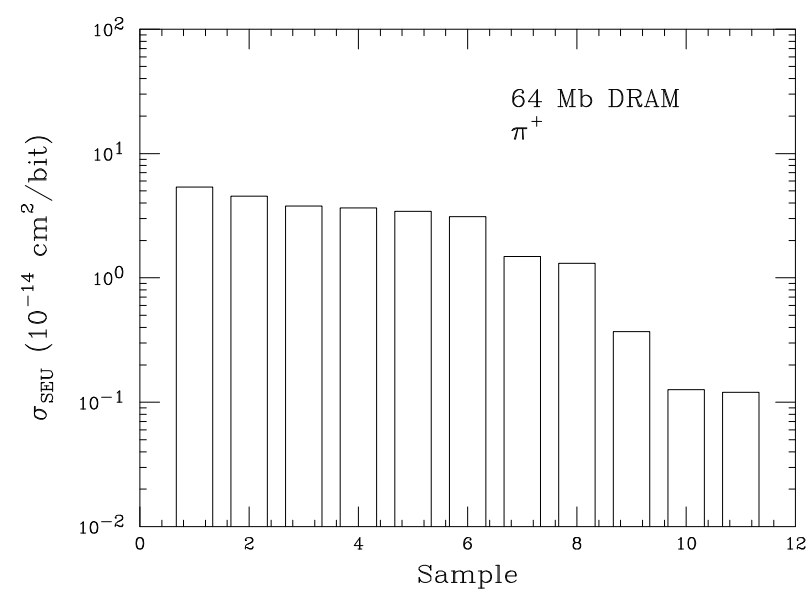

Figure 3. SEU cross sections for $154 \mathrm{MeV} \pi^{+}$are shown for a range of $64 \mathrm{Mb}$ samples. The lowest values are for TIC samples.
Only SC and TIC technologies were used for the $64 \mathrm{Mb}$ samples we tested, and again a strong difference is found for cross sections for these technologies. Cross sections for several samples from several manufacturers are shown in Fig. 3.

A similar pion experiment measured SEU in a commercial $64 \mathrm{~kb}$ SRAM, with results shown in Fig. 4. Largest pion SEU cross sections for $16 \mathrm{Mb}$ and $64 \mathrm{Mb}$ DRAM samples were $6 \times 10^{-13}$ and $4 \times 10^{-14} \mathrm{~cm}^{2}$, comparable to these for the SRAM sample.

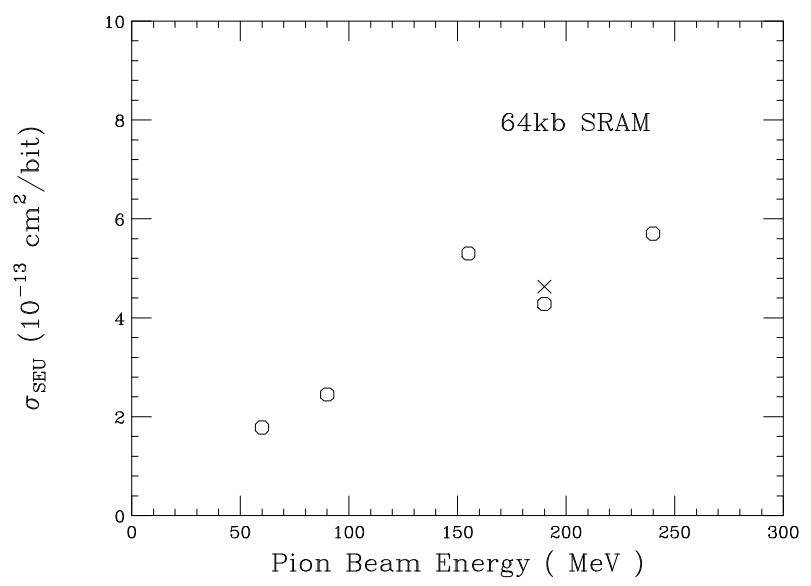

Figure 4. SEU cross sections in a $64 \mathrm{~kb}$ SRAM sample are shown by circles for a range of $\pi^{+}$beam energies, and a single $\pi^{-}$datum is shown by the cross.

To understand these effects, we need to compare the charge left in Si by ionizing particles to the charge that determines a memory state. The critical charge that distinguishes a ' 0 ' from a ' 1 ' in a modern device is about $50 \mathrm{fC}$ or about 0.3 million ion pairs. One $\mathrm{MeV}$ deposited in $\mathrm{Si}$ creates 0.28 million pairs, or $45 \mathrm{fC}$, very near a critical charge. A minimum ionizing particle deposits only about $0.47 \mathrm{keV}$ per micron, or 130 pairs per micron of its passage. Since memory cells are only a few microns on a side, this $\mathrm{dE} / \mathrm{dx}$ is not the cause of errors. In contrast the $5 \mathrm{MeV}$ alpha particles from natural radioactivity have a range of 25 microns, and deposit hundreds of keV per micron as they slow. If charge is collected from different volumes of Si by the several chip types, greater or lesser amounts of charge may be collected.

The set of identical $16 \mathrm{Mb}$ samples used for Fig. 2 was studied by us with the same equipment for $148 \mathrm{MeV}$ protons, $150 \mathrm{MeV}$ pions and $14 \mathrm{MeV}$ neutrons.[4] Pions of this energy sit atop the fundamental 3-3 resonance where reaction cross sections are largest. The scatter plot to compare these is shown in Fig. 5. Note that the resonant energy pions have cross sections larger than for protons or neutrons.

With a similar set of identical $64 \mathrm{Mb}$ chip samples, we made a similar close comparison of SEU for $148 \mathrm{MeV}$ protons, $14 \mathrm{MeV}$ neutrons and $154 \mathrm{MeV}$ pions. The scatter plot for 14 different $64 \mathrm{Mb}$ chips is shown in Fig. 6. These pion data were also shown in Fig. 3. 


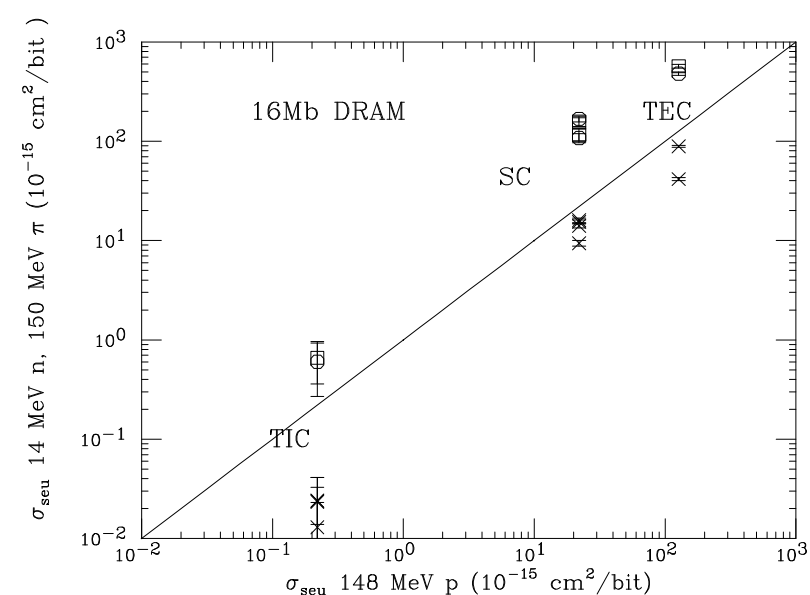

Figure 5. SEU cross sections for $16 \mathrm{Mb}$ samples are compared for $150 \mathrm{MeV}$ pi mesons and $148 \mathrm{MeV}$ protons. Circles compare $\pi^{+}$and protons and squares compare $\pi^{-}$and protons. Crosses compare SEU cross sections for $14 \mathrm{MeV}$ neutrons to those for 148 $\mathrm{MeV}$ protons. The same chip was used for each datum, with TIC technology for the cluster of low cross sections. The line indicates equal cross sections for each beam species.

In both scatter plots the solid line indicates proton cross sections equal to those for the neutrons or the pions. At this resonance energy pion $\sigma_{S E U}$ are about three times larger than proton data. We note the wide range of cross sections, and thus susceptibility to errors in the circuits, with TIC devices less sensitive than SC devices. The TEC technology is not used for $64 \mathrm{Mb}$ chips.

One might expect $\sigma_{S E U}$ to be related to $\sigma_{R}$, the reaction cross section for all that happens other than elastic scattering. These $\sigma_{R}$ are known or calculated reliably. Pion $\sigma_{S E U}$ for $16 \mathrm{Mb}$ TEC devices show a close proportionality to $\sigma_{R}$ from 50 to $400 \mathrm{MeV}$, across the important resonance region, as shown in Fig. 1. This ratio is very different for other samples, but the resonance is visible in all samples studied for a range of pion beam energies. The ratio of proton $\sigma_{S E U}$ to $\sigma_{R}$ increases only slightly from 60 to $300 \mathrm{MeV}$.[6]

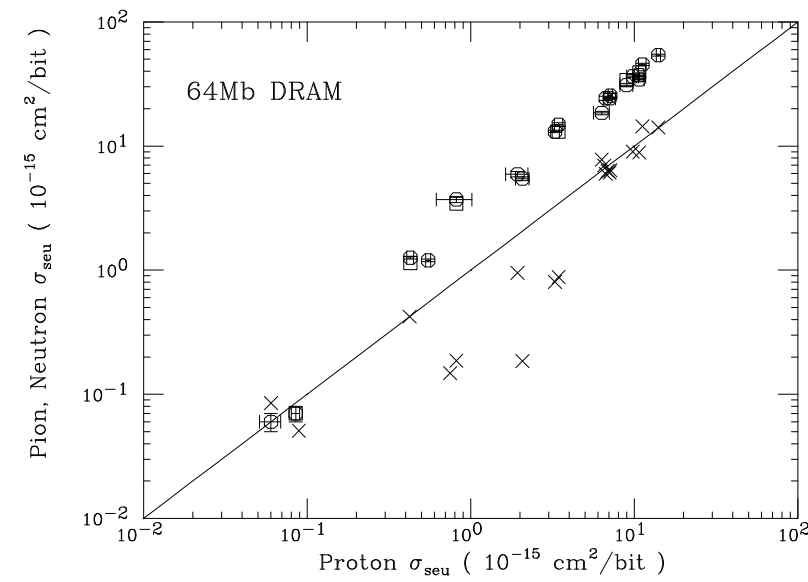

Figure 6. SEU cross sections for $64 \mathrm{Mb}$ samples are compared for $150 \mathrm{MeV}$ pi mesons and $148 \mathrm{MeV}$ protons. Circles compare $\pi^{+}$and protons and squares compare $\pi^{-}$and protons. Crosses compare SEU cross sections for $14 \mathrm{MeV}$ neutrons to those for 148 $\mathrm{MeV}$ protons. The same chip was used for each datum, with TIC technology for the cluster of low cross sections. The line indicates equal cross sections for each beam species.
These data show smaller SEU cross sections for $64 \mathrm{Mb}$ samples than for $16 \mathrm{Mb}$ samples. If the chip area is the same, there would be a factor of four difference between the cross-sectional areas per bit for these two densities. Older chips with lower densities have also had soft error cross sections measured for cosmic rays.[10] Fig. 7 shows those SEU cross sections and those currently measured for pions with the largest cross sections. These cross sections drop more quickly than just the cross sectional area, and more like the linear dimension to the third power, as if the sensitive volume is decreasing, not just the cross sectional area.

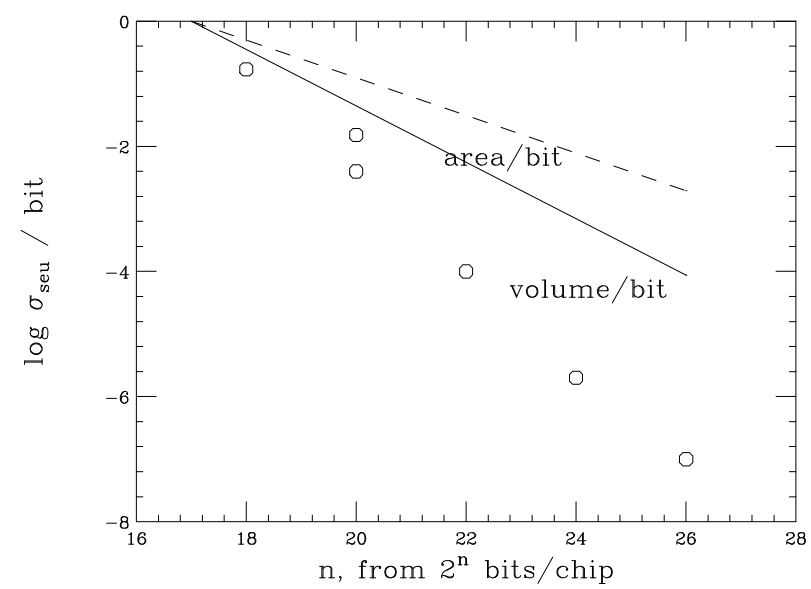

Figure 7. SEU cross sections per bit have been decreasing with increasing bit density. Present pion data for the examples with largest cross sections for $16 \mathrm{Mb}$ and $64 \mathrm{Mb}(\mathrm{n}=26)$ are compared to earlier cosmic ray data. [3] The decrease is stronger than expected even by the decreasing square of linear dimensions.

For these typical modern devices, we have thus found empirical connections among data for neutrons, protons and pions. These suffice for modeling responses to cosmic ray fluxes for many current applications. Further, our results indicate that we need measure $\sigma_{S E U}$ for only one set of these beams to infer responses to the others. This can lead to a dramatic decrease in the cost of testing.

Experts can model the fluxes of protons, neutrons and pions from cosmic rays at a range of altitudes and latitudes. Together with our data, we can now calculate rates of soft errors in complex avionics packages for commercial and military aircraft, depending on the type and manufacturer of the memory chips used.[11]

\section{Reaction mechanisms}

This empirical knowledge of chip SEU cross sections should be based upon an understanding of the means by which lighly ionizing protons, neutrons and pions interact to deposit charge within devices. Extensive modeling studies have been carried out for many electonic components by those in the industry.[12] Because of their high rate of energy loss, low energy alpha particle production is a likely direct means to induce errors, since it has long been known that $5 \mathrm{MeV}$ alpha particles from natural radioactivity are 
dangerous sources of SEU.[1] Reaction model calculations for protons and pions on Si provide spectra for emerging alpha particles that peak near this energy of $5 \mathrm{MeV}$ [13], but measurements have not gone to such low energies.

We have carried out an early measurement of alpha particles in the $5 \mathrm{MeV}$ energy range emerging from $\mathrm{Al}$, as a simple substitute for $\mathrm{Si}$, using pion beams from TRIUMF and commercial plastic track detector technology. Error bars are large, and experimental control was difficult. Our method was also not able to distinguish between alpha particles and low energy protons which also leave heavy ionization in the material. Our method has no real energy determination, but is expected to be sensitive for alpha particle energies from 0.5 to $6 \mathrm{MeV}$.

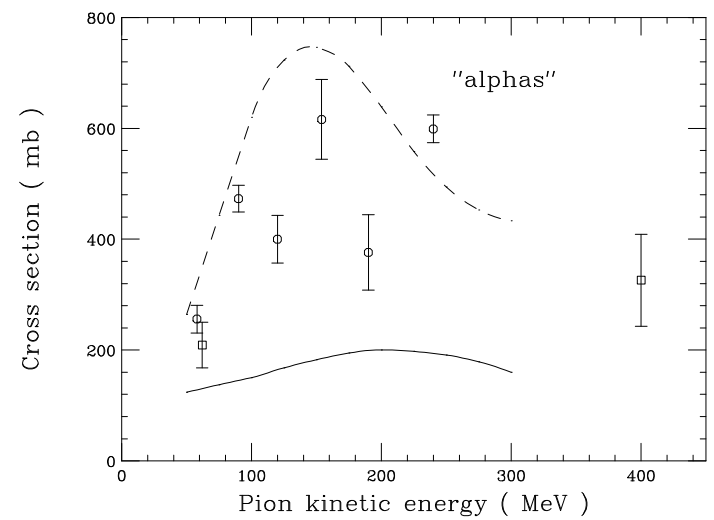

Figure 8. Squares show data for $\pi^{-}$induced yields of alpha particles from 0.5 to $6 \mathrm{MeV}$ from an $\mathrm{Al}$ sample, while circles show $\pi^{+}$data. These data used plastic track detector technology, without the ability to distinguish energies or to preclude events due to low energy protons. The solid curve shows the integrals over computed alpha particle spectra across the range of our expected sensitivity.[14] The dashed curve shows the computed pion reaction cross sections for ${ }^{27} \mathrm{Al}$.

Nonetheless, we present in Fig. 8 the cross sections we have measured.[14] Comparison is made to an integral over the calculated spectra [13] across the alpha particle energy range we expect to be able to sense, and to the computed reaction cross sections. Yields are large and seem to reflect the 3-3 resonance as expected. The measured cross sections are near the reaction cross sections, indicating a multiplicity greater than one, possible for the very high excitation energies that can be available from pion absorption. We are thus confident, even from this rough measurement, that pion (and presumably proton and high energy neutron) reactions produce alpha particles within the body of the microcircuits, and these short range and heavily ionizing products deposit the energy and charge needed to induce a transition among ones and zeroes to cause the SEU.

\section{Acknowledgements}

This work was supported in part by the USDOE, with the strong collaboration of IBM and the US Naval Academy.

\section{References}

[1] T. May and N. Woods, IEEE Trans. Electron Devices 26, 2 (1979).

[2] J. F. Ziegler and W. A. Lanford, Science 200, 776 (1979).

[3] J. F. Ziegler et al., IBM J. Research and Devel. 40, 3 (1996).

[4] J. F. Ziegler et al., IEEE Trans. Solid State Circuits 33, 246 (1997).

[5] C. J. Gelderloos et al., IEEE Trans. Nucl . Sci. 44, 2237 (1997).

[6] G. J. Hofman et al., IEEE Trans. Nucl. Sci. 47, 403 (2000).

[7] J. D. Shell et al., 1997 HEART/GOMAC Conference, Las Vegas.

[8] A. A. Ebrahim and R. J. Peterson, Phys. Rev. C54, 2499 (1996); R. J. Peterson, Few Body Sys. Suppl. 9, 17 (1995).

[9] R. F. Carlson, At. Data Nucl. Data Tables 63, 93 (1996).

[10] cited in Ref. 3.

[11] M. E. Nelson et al., Jour. Radiation Effects, Research and Engin.19, 100 (2001).

[12] G. R. Srinivasan, IEEE Trans. Nucl. Sci.41, 2063 (1994).

[13] G. Srinavasan, H. Tang and P. Murley, IEEE Trans. Nucl. Sci. 41, 2083 (1994); H. Tang, IBM Jour. Res. Dev.40, 91 (1996).

[14] R. J. Peterson et al., Radiation Measurements 35, 565 (2002). 